\title{
Voltage Dip Detection in noisy Environments using Tracking Filters
}

\author{
Florin Molnar-Matei \\ Politehnica University of Timisoara, 720229, Romania \\ florin.molnar@et.upt.ro
}

Keywords: Power Quality, Voltage Dips, Digital Signal Processing, Tracking Filters, Noise.

\begin{abstract}
Due of its significant economical impact, voltage dip analysis is an important domain today. Monitoring these phenomena (also called sags) require powerful techniques. Digital Signal Processing (DSP) algorithms are currently employed to fulfill this task. One of the possible algorithms is the $\alpha-\beta-\gamma$ filter. Discovered and tested during the cold war, this algorithm can estimate a parameter of interest one-step-ahead based on the previously observed values. In previous work, this filter revealed very good performance in the absence of noise. However, noise is present in every technical environment. This paper examines the dip detection performance using the above mentioned tracking filter in a noisy environment.
\end{abstract}

\section{Introduction}

The electricity demands increase as the society evolves. On one hand, the population has increased. On the other hand their needs have grown. As such, the industrial development which is expected to support people needs has demanded large power networks.

Power-plants, transformers, and high-voltage lines are elements that form the power systems today. A major concern today for many industrial customers is the so-called Power Quality (PQ). Several categories of events can occur in these power networks during every-day operation. Clearly, the toughest possible event is the short circuit (this may result in total power disruption).

Another event is the so-called voltage reductions. They may be caused by short circuits (they care quite severe in these cases) or by varying loads [1]. These phenomena are known as "voltage dips" (or "voltage sags") in the PQ domain. Simply said, they are Root-Mean-Square (RMS) voltage variations (considering three-phase systems) which affect the industrial consumers. According to Gallo [2], these events end due to the automatic switching, load stabilization and/or power system actions. However, they are considered important issues [3].

Standards define voltage dips as one of the most important aspects of power quality [4]. Common ways to characterizing these phenomena have to be established. They are considered of rectangular shape, characterized by magnitude and duration [4]. Commonly, the dip's magnitude is defined to be the voltage reduction under the $90 \%$ threshold [4]. If the voltage falls below $10 \%$, the perturbation is considered to be an interruption. A typical voltage dip form can be seen in Fig. 1. The duration of the event is the time measured from the moment when RMS voltage drops below the $90 \%$ threshold to the moment it rises above it. Possible values range from few power cycles (tens of milliseconds) to one minute. Balanced dips are many times assumed in three-phase power systems. In reality, most voltage dips occur due to unbalanced faults, which introduce different values of voltage for each phase and phase angle shift [1].

The IEC Standard [5] describes a procedure for dip detection. According to this procedure, the RMS voltage is compared against a threshold (usually a percentage) which is typically $90 \%$ of the nominal RMS. One cycle of acquired data is used to compute the RMS voltage. The IEC standard defines the RMS voltage to be the root mean square value of the sinusoidal wave measured over one cycle and commencing at zero-crossing. Unfortunately, the zero-crossing moments are not reliable during a voltage dip. 


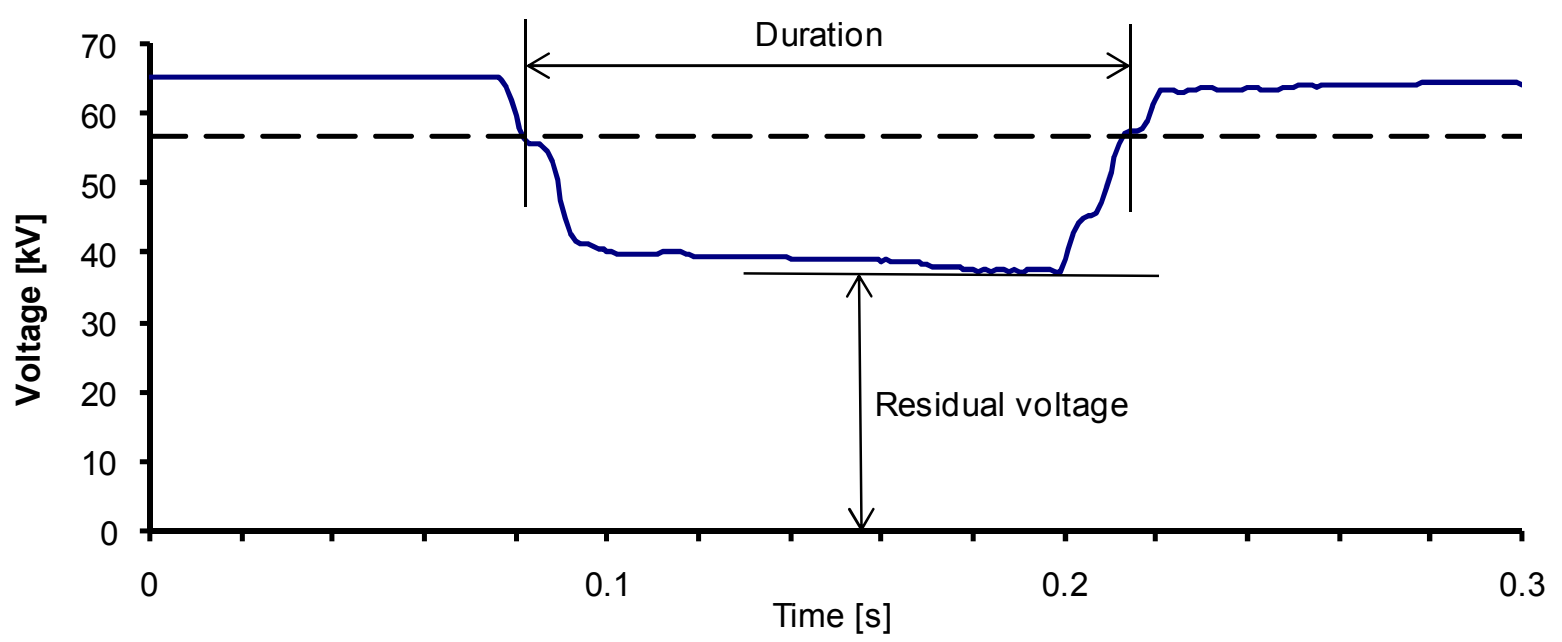

Fig.1. Tipical voltage dip in $110 \mathrm{kV}$ network

Two problems have to be addressed for dip characterization: 1) to establish which voltages will be measured - phase to phase or phase to neutral and 2) to establish the DSP technologies to be employed. Didden [6] and Leborgne et al [7] have studied these connections for voltage measurement. Despite the fact that the two articles have different purpose, they underline pros and cons for both voltage measurement connections. However, since most of the recorders are connected between phase and neutral, it is recommended to use this connection type.

In literature, there are many DSP technologies employed for voltage dip detection. Many of them use different forms of wavelet transforms or Kalman filters [3], [8]. Literature analysis reveals that wavelet transform is preferred when the dips are targeted together with other PQ problems.

Styvaktalcis et al [9] performed a comparative study in using four methods for RMS computation: the RMS value calculated over one-cycle overlapping windows, the RMS value calculated over half-cycle overlapping windows, Kalman filter of order 20 and Kalman filter order 1 cascaded with a low-pass filter. They conclude that the speed of detection depends for each method on the voltage dip magnitude, the point where the dip starts (on the wave) and the phase angle jump that is caused. To monitor three-phase events Barros and Perez [3] have also proposed the Kalman filter. Three Kalman filters implemented on a DSP structure monitor the RMS voltages. These values are periodically calculated from sampling voltage values. Kalman filter proved a proper method for voltage dips automatic detection.

In 2004 the same Dash et. al [8] have extended the Kalman filter's use by combining it with the S-Transform. The filter was employed to extract data for event characterization while the STransform was needed for perturbation detection. A combination of discrete wavelet transform, artificial intelligence (fuzzy expert system) and Kalman was proposed in [10]. The discrete wavelet transform was used to improve the Signal-Noise Ratio (SNR) while the Kalman filter extracted the needed information. The fuzzy system was employed to analyze the data for power quality characterization.

A better RMS detection was proposed by Reddy in [11] by using an Unscented Kalman filter combined with artificial intelligence techniques for fast tracking of the power quality disturbance. The authors compare the algorithm with the Extended Kalman filter and demonstrate the superiority of their results.

During the sudden RMS reduction, the dip distorts the sinusoidal wave, therefore introducing harmonics. Santoso et al [12] has proposed a dyadic-orthonormal wavelet transform for PQ disturbances. Their key idea was to decompose the disturbance into other signals using the MultiResolution Signal Decomposition technique. The wavelet transform proved to be a powerful tool. However, the mother wavelet selection played an important role in detecting disturbance.

An alternative detection algorithm based on the Generalized Likelihood Ratio Test (GLRT) was proposed by Guerrieri et al in [13]. Their approach can achieve competitive statistical performance when smooth transients are associated to the voltage dips. 
Oftentimes, the voltage dip detectors are built using embedded hardware (DSP or microcontroller-based). When operating, its main tasks include data acquisitions and analog to digital conversion, RMS calculation (several previously-sampled voltage values stored in the memory are used), signal processing for dip detection and eventually, recording the event (for log files) and alert issuing. Since the dip can occur on any phase, a three-phase watching is indicated. However, in such a case the amount of work triples. A DSP board (which offers a significant computation power) is preferred (instead of a microcontroller) for this task [3]. As such, any possible algorithm simplification or increase in computational power is therefore useful.

In the literature discussed above, there is no discussion regarding the noise in the measuring environment. The authors did not test the DSP involved in noisy environment. Therefore, the performance is not known. Introduced before [14], the $\alpha-\beta-\gamma$ filter has the advantage of being simpler (requiring a lower amount of computation which in case of a three-phase system triples) than the Kalman filter. In [14] it was proved that this filter can be tuned to detect dips faster than Kalman. In this paper, $\alpha-\beta-\gamma$ filter is going to be tested in a noisy environment. The objective of this paper is to assess its performance in a noisy environment by comparing the results against the ones delivered by the same filter in a noiseless environment. Section II of this paper introduces this family of filters and describes the $\alpha-\beta-\gamma$ filter. Section III discusses the filter's stability using the Jury' stability criterion (presented as well). Section IV presents experiments in a noisy environment for a $110 \mathrm{kV}$ power line and discusses the results.

\section{The $\alpha-\beta-\gamma$ Tracking Filter}

The recent technologies have brought significant transformations to our life. Indeed, about a hundred microprocessors can be found today a modern home. Numerous real-life applications use digital data today. It is almost impossible to imagine a fully-analog device today.

Civilian and military applications such as the air-traffic handling, the missile interception and the anti-submarine warfare require the use of discrete-time data to predict the kinematics of a moving object. The use of passive sonobuoys which have limited power capacity pushes for implementation of computationally inexpensive target-trackers.

The $\alpha-\beta$ family of filters was developed in the cold-war era by Sklansky [15]. At that time, it was intended for radar target tracking, position and velocity estimation from noisy measurements of range and bearing. Since then, they have been used for both predictions and tracking. Kalata and Murphy [16] have tried to use them for tracking with rate variations. Tenne and Singh [17] have studied ways to design them for optimal performance. In their work they also indicate how to select the parameters to obtain a stable filter. Corke and Good ['8] have compared their performance with Kalman filters in the computer vision domain. They have underlined that in case of using Kalman filters the coefficients will converge to constant values. Therefore, in their case the $\alpha-\beta-\gamma$ filters delivered similar performance with less computational effort.

Stanciu and Oh [19] have employed these filters in visual servoing to predict one-step-ahead the target's position in their image plane. Taking advantage of a feedforward controller this method has tremendously improved the tracking performance of their system. Stanciu and Sorandaru [20] have successfully tested this filter to forecast the energy-demand. Their implementation demonstrated a prediction error below $4 \%$.

There are two members in this family of algorithms. Both of them are working similarly. There are two steps: prediction and correction. The $\alpha-\beta$ filter is the simplest one which predicts the position only. The filter examined in this work is the extended version in this family called the $\alpha-\beta-$ $\gamma$ filter. In the prediction step, the algorithm estimates one-step-ahead the position (represented by the RMS voltage) and velocity (represented by the voltage gradient). In the correction step the filter is smoothing the position, the velocity and the acceleration, thus improving the tracking performance. The predicted values position Eq. 1 and the velocity Eq. 2 for iteration $k+1$ are written as functions of the current smoothed values for position, velocity, acceleration, and the sampling time $T$. 


$$
\begin{aligned}
& x_{p}(k+1)=x_{s}(k)+T \cdot v_{s}(k)+\frac{T^{2}}{2} \cdot a_{s}(k) \\
& v_{p}(k+1)=v_{s}(k)+T \cdot a_{s}(k)
\end{aligned}
$$

The position, the velocity and the acceleration are then corrected (smoothed) based on the observed position $x_{0}(k)$ at iteration $k$ in Eq.3, Eq.4 and Eq.5.

$$
\begin{aligned}
& x_{s}(k)=x_{p}(k)+\alpha \cdot\left[x_{o}(k)-x_{p}(k)\right] \\
& v_{s}(k)=v_{p}(k)+\frac{\beta}{T} \cdot\left[x_{o}(k)-x_{p}(k)\right] \\
& a_{s}(k)=a_{s}(k-1)+\left(\frac{\gamma}{2 \cdot T^{2}}\right) \cdot\left[x_{o}(k)-x_{p}(k)\right]
\end{aligned}
$$

\section{The Tracking Filter's Stability Analysis}

Prior to its implementation a stability study was performed. The objective was to determine the parameter ranges (for $\alpha, \beta$, and $\gamma$ ) which ensure a stable filter. To obtain the transfer function (which relates the two positions - predicted and observed) one has to apply the Z-transform to the prediction and the correction equations. After performing all calculations [14], the transfer function is given by (6) (where "P" stands for position):

$$
G_{P \alpha \beta \gamma}(z)=\frac{X_{p}(z)}{X_{0}(z)}=\frac{\left(\alpha+\beta+\frac{\gamma}{4}\right) \cdot z^{2}+\left(-2 \cdot \alpha-\beta+\frac{\gamma}{4}\right) \cdot z+\alpha}{z^{3}+\left(\alpha+\beta+\frac{\gamma}{4}-3\right) \cdot z^{2}+\left(-2 \cdot \alpha-\beta+\frac{\gamma}{4}+3\right) \cdot z+\alpha-1}
$$

For voltage dip detection, the predicted "position" is assumed to be the RMS voltage at next iteration. Another transfer function relating the predicted "velocity" to the observed position can be derived. However, the voltage rate of change (which corresponds to the "velocity" parameter of the tracking filter) was not examined in this work.

A stability criterion is needed to examine the filter stability. Since the z-plane's boundary is different that of the s-plane, the Routh-Hurwitz stability criterion cannot be applied directly. However, a similar method but for discrete systems is represented by the Jury's stability test. This is used in the following to study the filter's stability. A table is constructed based on the coefficients of the characteristic polynomials (Table I).

Table I. The Jury's Stability Test Table for the Filter

\begin{tabular}{|c|c|c|c|}
\hline $\mathrm{Z}^{0}$ & $\mathrm{Z}^{1}$ & $\mathrm{Z}^{2}$ & $\mathrm{Z}^{3}$ \\
\hline$\alpha-1$ & $-2 \cdot \alpha-\beta+\frac{\gamma}{4}+3$ & $\alpha+\beta+\frac{\gamma}{4}-3$ & 1 \\
\hline 1 & $\alpha+\beta+\frac{\gamma}{4}-3$ & $-2 \cdot \alpha+\beta+\frac{\gamma}{4}+3$ & $\alpha-1$ \\
\hline$\alpha(\alpha-2)$ & $\alpha\left(4-2 \cdot \alpha-\beta+\frac{\gamma}{4}\right)-\frac{1}{2}$ & $\alpha\left(\alpha+\beta-2+\frac{\gamma}{4}\right)-\frac{1}{2}$ & \\
\hline
\end{tabular}

According to this theory there are two necessary stability conditions and three sufficient stability conditions. For the first necessary stability condition, is then:

$$
1+\alpha+\beta+\frac{\gamma}{4}-3-2 \cdot \alpha-\beta+\frac{\gamma}{4}+3+\alpha-1=\frac{\gamma}{2}>0
$$

Equation (7) simply restricts the parameter $\gamma$ to positive values. The second necessary stability condition translates to:

$$
2 \cdot \alpha+\beta<4
$$


There are two sufficient stability conditions to be fulfilled. The first is:

$$
|\alpha-1|<1
$$

This is equivalent to $0<\alpha<2$. The second condition which ensures stability is:

$$
|\alpha \cdot(\alpha-2)|>\left|\alpha \cdot(\alpha-2)+\alpha \cdot\left(\beta+\frac{\gamma}{4}\right)-\frac{\gamma}{2}\right|
$$

The parameter $\alpha$ is always positive and the quantity $(\alpha-2)$ is always negative. This leads to:

$$
\alpha \cdot\left(\beta+\frac{\gamma}{4}\right)-\frac{\gamma}{2}>0
$$

Equation (11) translates to:

$$
\gamma<\frac{4 \cdot \alpha \cdot \beta}{2-\alpha}
$$

The implemented filter has coefficients which comply with the above restrictions in order to ensure its stability. The chosen parameters in the above intervals are $\alpha=0.52, \beta=0.7$, and $\gamma=0.28$. Small modification of these values did not reveal noticeable filter-behavior change.

\section{Simulations and Results}

The power voltage is sampled 20 times/cycle which results in a sampling frequency of $1000 \mathrm{~Hz}$. The RMS voltage is computed using (13).

$$
V_{R M S}(k)=\sqrt{\sum_{i=k-20}^{k}(v(i))^{2}}
$$

Here, $v(i)$ is the $\mathrm{i}^{\text {th }}$ sample (one of the last $20^{\text {th }}$ samples). The last 20 samples are used to compute the actual RMS voltage. Consequently, a three-phase system triples the work to be done.

The above described filter has been used to determine how fast estimate de start of voltage dip in two cases: noiseless and noisy environments. In this regard, several tests have been performed to assess the filter's behavior in different situations of disturbance. The noisy values have been obtained by adding to the initial waves randomly generated noises. Three significant noisy situations were chosen to present the filter's results against the ones obtained without disturbance.

For the noiseless case Fig. 2 shows the power grid's waves (recorded with $1 \mathrm{kHz}$ data acquisition system) on the three phases ( $\mathrm{R}$ - marked with $\mathrm{x}, \mathrm{S}$ - marked with circles, and $\mathrm{T}-$ marked with squares). Fig. 2 shows that the phase affected by dip is T. Fig. 4 presents the filter's behavior in the presence of the voltage dip and demonstrates its detection ability. The x-marked curve in Fig. 4 represents the filter's estimated values while the circle-marked curve represents the threshold. The dashed line represents the calculated RMS voltage using equation (13).

The first noisy situation assumed a random disturbance of 5\%. This mean that each recorded value $v(i)$, it was replaced with a random value generated in the range of $0.95^{*} v(i) \ldots 1.05^{*} v(i)$. Fig. 3 shows the power grid waves. Fig. 5 presents the filter's behavior. As it can be seen in this figure, the filter successfully detects the dip.

The next two cases assumed a $10 \%$ random disturbance in the power waves. This mean that each recorded value $v(i)$ was replaced with a random value generated in the range of $0.90^{*} v(i) \ldots$ $1.10^{*} v(i)$. The signals are presented in Fig. 6 (first case) and 7 (the second case). Such levels of noise have been considered because it was thought that both, successful dip-detection (case 1 - Fig. 8) and dip-detection failure (case 2 - Fig. 9), situations may exist. In this situation, the "dipdetection failure" means that the filter has a delay in voltage dip detection when compared with other cases. 


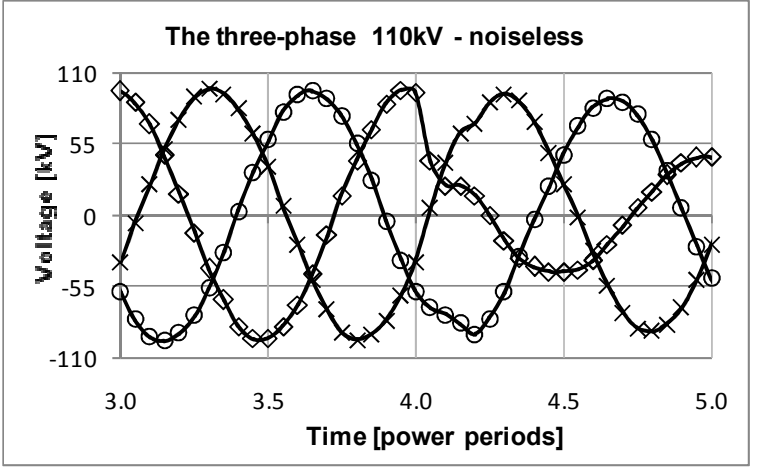

Fig.2. Three-phase power network voltage dip noiseless

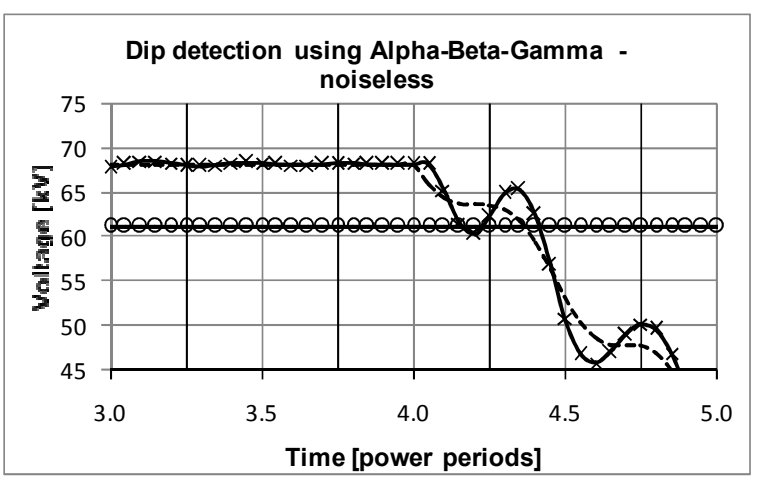

Fig.4. Three-phase power network voltage noiseless dip detection

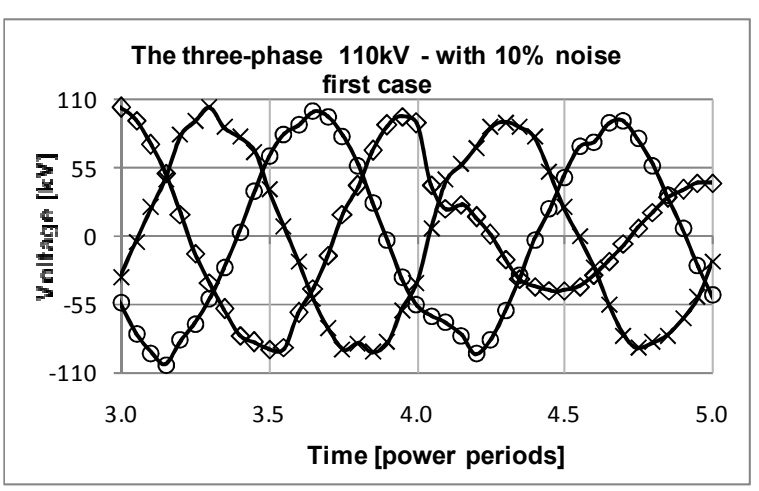

Fig.6. Three-phase power network voltage dip with $10 \%$ noise - first case

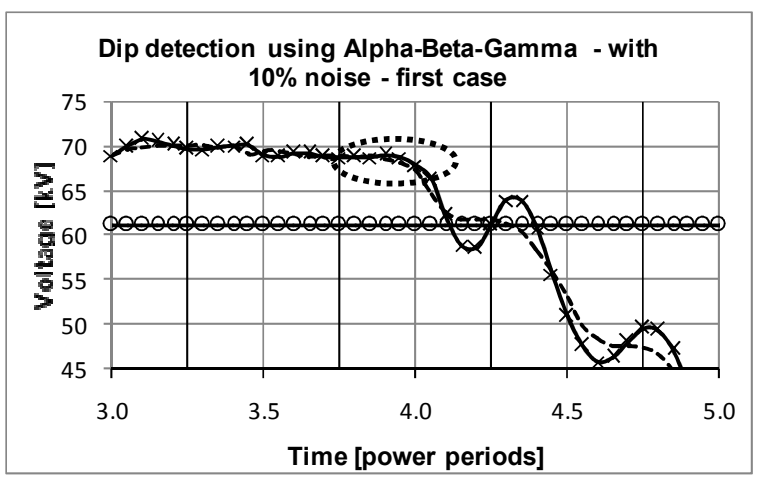

Fig.8. Three-phase power network voltage dip detection under $10 \%$ noise - first case

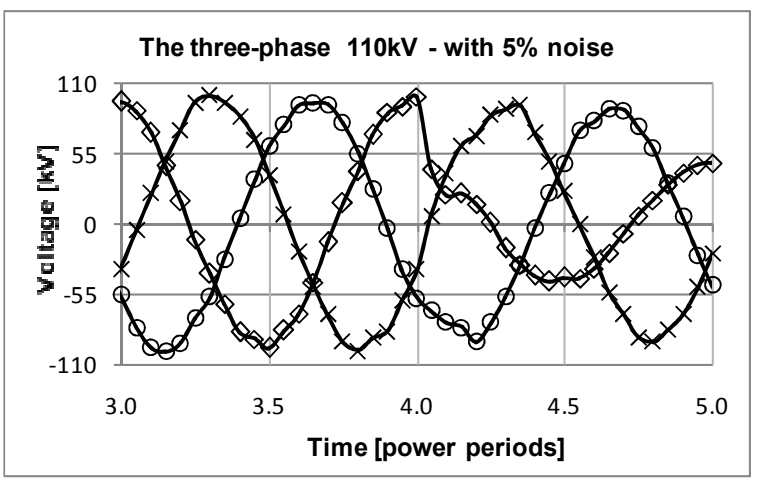

Fig.3. Three-phase power network voltage dip with $5 \%$ noise

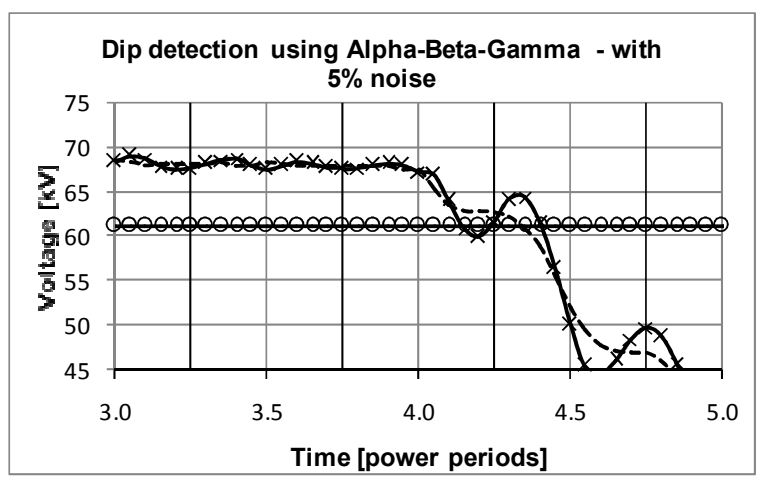

Fig.5. Three-phase power network voltage dip detection under 5\% noise

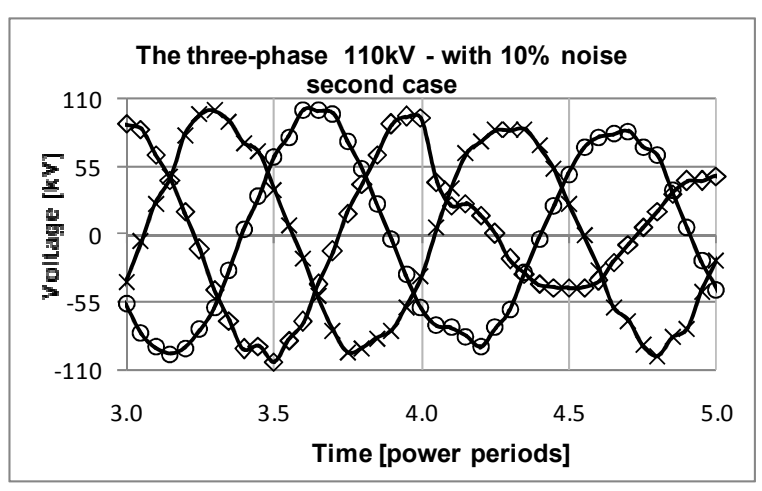

Fig.7. Three-phase power network voltage dip with $10 \%$ noise - second case

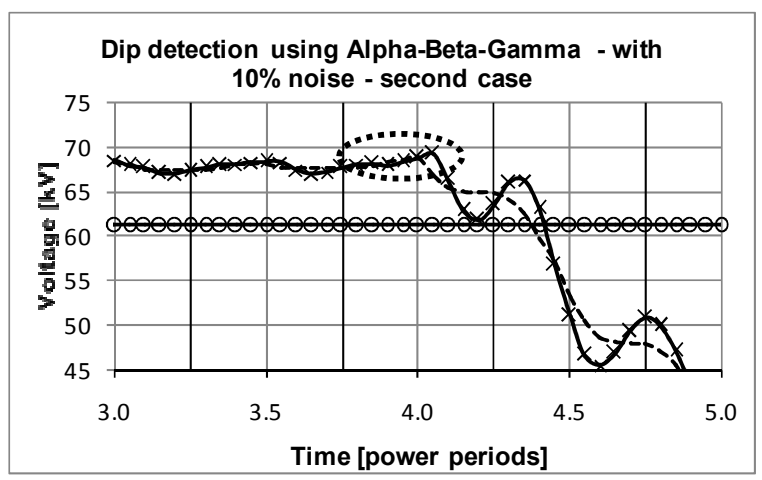

Fig.9. Three-phase power network voltage dip detection under $10 \%$ noise - second case 
The two cases presented were the extreme cases among a set of performed tests. An analysis was performed on all the results in order to identify the cause of the dip detection failure. Consequently, if the RMS value increases fast (due to the noise) the filter's estimation does not reach the threshold value. Still, its estimation is closed to it. To highlight this thing, the beginning of the dip has been marked with circles in Fig. 8 and Fig.9.

If taking into account that on $10 \%$ represents a huge disturbance which rarely occurs in day-today operation, one can conclude that $\alpha-\beta-\gamma$ filter works very well in the presence of disturbance.

\section{Conclusion and Future Work}

Voltage dips are undesired phenomena affecting the power quality. They are regarded to be the main issue in power quality today. Digital signal processing techniques like Kalman filters and Discrete Wavelet transforms were proposed to detect them.

To perform detection, the voltage is sampled by the digital system. The RMS value has to be computed using several sampled values. A detection algorithm has to be employed to detect these events. If this computation has to be done for a three-phase system, the amount of work triples. Consequently, any computation reduction is useful.

This paper proposes assess the behavior of the $\alpha-\beta-\gamma$ filter in a noisy environment. The results reveal the filter's ability to work even in the presents of significant noise. Still, for values of disturbance of $10 \%$, a failure to detect the dip situation has been found. However, $10 \%$ disturbance for a $110 \mathrm{KV}$ power line rarely occurs in the day-to-day operation. This emphasizes a good behavior of the filter even in the presence of noise.

In this paper, the coefficients which comply with the restrictions of the algorithm have been empirically chosen. However, future work will have to examine whether there is a correlation between the filter's coefficients and voltage dip's or network parameters.

\section{Acknowledgement}

This paper was supported by the project "Development and support for multidisciplinary postdoctoral programs in major technical areas of national strategy for Research - Development Innovation" 4D-POSTDOC, contract nr. POSDRU/89/1.5/S/52603, project co-funded by the European Social Fund through Sectorial Operational Program Human Resources Development 2007-2013.

\section{References}

[1] M.H.J. Bollen, Understanding Power Quality Problems. Voltage Sags and Interruptions., NY: IEEE Press, New York, 2000,

[2] D. Gallo, C. Landi, M. Luiso, Accuracy Analysis of Algorithms Adopted in Voltage Dip Measurements, IEEE Trans. on Instrumentation and Measurement, Vol. 59, No. 10, (2010) 2652-2659.

[3] J. Barros, E. Perez, Automatic Detection and Analysis of Voltage Events in power Systems, IEEE Trans. on Instrumentation and Measurement, Vol. 55, No. 5, (2006) 1487-1493.

[4] Voltage Characteristics of Electricity Supplied by Public Distribution Networks, Standard EN 50160 (2007).

[5] Electromagnetic Compatibility (EMC) Part 4-30: Testing and Measurement Techniques Power Quality Measurement Methods, IEC 61000-4-30 (2003).

[6] M. Didden, E. De Jaeger, W. D’Haeseleer, R. Belmans, How to connect a voltage sag measuring device: Phase to phase or phase to neutral?, IEEE Trans. on Power Delivery, vol. 20, no. 2 (2005) 1174-1181.

[7] R.C. Leborgne, G. Olguin, M.H.J. Bollen, The Influence of PQ-Monitor Connection on Voltage Dip Measurements, Proceedings of IEE MedPower Conference, Cyprus (2004). 
[8] P.K. Dash, M.V. Chilukuri, Hybrid S-Transform and Kalman Filtering Approach for Detection and Measurement of Short Duration Disturbances in Power Networks, IEEE Trans. on Instrumentation and Measurement, Vol. 53, No. 2 (2004) 588-596.

[9] E. Styvaktalcis, I.Y. Gu, M.H.J. Bollen, Voltage Dip Detection and Power System Transients, Power Engineering Society Summer Meeting, Vancouver, Canada, 15-19 Jul. (2001) 683-688.

[10] A.A. Abdelsalam, A.A. Eldesouky, A.A. Sallam, Characterization of power quality disturbances using hybrid technique of linear Kalman filter and fuzzy-expert system, Electric Power System Research, vol. 83 (2011) 41-50.

[11] J.B.V. Reddy, P.K. Dash, R. Samantaray, A.K. Moharana, Fast Tracking of Power Quality Disturbance Signals Using an Optimized Unscented Filter, IEEE Trans. on Instrumentation and Measurement, Vol. 58, No. 12 (2009) 3943-3952.

[12] S. Santoso, E.J. Powers W.M. Grady, P. Hofman, Power quality assessment via wavelets transform analysis, IEEE Trans. on Power Delivery, Vol. 11, No. 2 (1996) 924-930.

[13] G. Guerrieri, A. Moschitta, P. Carbone, C. Muscas, Statistical Properties of Voltage Dip Detectors, IEEE Trans. on Instrumentation and Measurement, Vol. 59, No. 11 (2010) 28002807.

[14] I.R. Stanciu, F. Molnar-Matei, Detecting Power Voltage Dips using Tracking Filters: A comparison against Kalman, in Advances in Electrical and Computer Engineering Journal, vol. 12, no. 4 (2012) 77-82, 2012.

[15] J. Sklansky, Optimizing the dynamic parameter of a track-while-scan system, RCA Laboratories, Princeton, NJ, 1957.

[16] P.R. Kalata, K.M. Murphy, $\alpha-\beta$ target tracking with track rate variations, Proceedings of $29^{\text {th }}$ Southeastern Symposium on System Theory (1997) 70-74.

[17] D. Tenne, T. Singh, Optimal design of $\alpha-\beta-(\gamma)$ filters, Proceedings of American Control Conference, Chicago, Illinois, vol. 6 (2000) 4348-4352.

[18] P.I. Corke, M.C. Good, Dynamic effects in high-performance visual servoing, International Conference on Robotics and Automation, Nice, France, (1992) 1838-1843.

[19] R. Stanciu, P.Y. Oh, Human-in-the-loop Camera Control for a Mechatronic Broadcast Boom, IEEE/ASME Transactions on Mechatronics, Vol. 12, Issue 1 (2007) 41-52.

[20] I.R. Stanciu, C. Sorandaru, Low-cost, short-term electric load prediction using the $\alpha-\beta-\gamma$ filter, $15^{\text {th }}$ IEEE International Conference on Intelligent Engineering Systems (INES), Poprad, Slovakia, (2011) 335-340. 